\section{What's mice got to do with it?}

\author{
Kenneth W. Kinzler and Bert Vogelstein
}

LESS than two years ago, a fiercely competitive race to find one of the genes responsible for hereditary breast cancer ended with the discovery of $B R C A 1$ (ref. 1). As is not uncommon, the sequence of the new gene provided few insights into its function. Thus began a new race to define the function of the gene product, a race that has turned out to be even more competitive and controversial than the last.

Among the newest entries in this contest are mouse 'knockouts' devoid of the $B R C A 1$ gene, which are described in Nature Genetics by Gowen et al. ${ }^{2}$ and in Cell by Hakem et al. ${ }^{3}$. Somewhat surprisingly, $B R C A 1$-null mice die during early embryogenesis, but they have already provided some new clues consistent with the idea that the BRCA1 protein is a transcription factor that puts the brakes on cell growth. This proposal is neatly supported by results from Chapman and Verma, reported on page 678 of this issue $^{4}$, which reveal that the carboxy terminus of BRCA 1 contains a domain that can activate transcription.

The embryonic death of the homozygous knockout mice was unexpected because humans with homozygous mutations of BRCA1 are capable of normal growth and development, with their only defect being a predisposition to breast and ovarian cancer similar to that found in heterozygotes 5 . In contrast, the heterozygous mice have no apparent predisposition to breast cancer (at least by one year of age; refs 2 and 3, and T. Mak, personal communication). Although residual activity of alternatively spliced forms of $B R C A 1 \mathrm{might}$ account for the viability of human homozygous mutants, the most obvious implication of these results is that Mus musculus and Homo sapiens are not the same beasts, a detail that often seems more obvious to our children than to us 'professionals'. Previous knockouts of cancer-related genes have, in fact, usually revealed phenotypes in mice different from those expected from the analogous humans. Some tumour-suppressor-gene knockouts in mice result in no tumour predisposition (for example, $W T 1$ ), whereas many others result in tumours of cell types that are significantly different from those observed in humans with inherited mutations of the same genes.

In the light of these substantial differences, are studies of mouse knockouts useless, or worse, misleading, for our understanding of human cancer? Certainly not. Although the series of events resulting in phenotypes as complex as neoplasia cannot be expected to be equivalent in mice and humans, the interactions between individual gene products and the biochemical pathways connecting them are likely to be well conserved. Murine cells with disrupted genes thus offer unique opportunities for studying such pathways in a highly controlled fashion.

Mice with disrupted p53 genes, for example, have provided unequivocal evidence that p53 protein is required for the normal response to agents that induce cell-cycle perturbations, a hypothesis that could only be suboptimally tested before the creation of these mice. In mouse cells without $B R C A 1$, there are substantial changes in the expression of the p53regulated genes $p 21$ and $m d m 2$ (ref. 3). This connection between the pathways controlled by BRCA1 and p53 is supported by independent studies demonstrating a striking coincidence between the cell-cycle distributions of BRCA1 and p53 proteins ${ }^{6,7}$. Interestingly, inherited mutations of p53 result in a predisposition to breast cancer in humans (but not in mice!)

How do the observations in $B R C A 1$-null mice jive with previous studies of BRCA1 function in human cells? Some recent observations support the idea that BRCA1, like p53, is a transcription factor, but the data are somewhat contradictory. Most have focused on the intracellular localization of the gene product. The first clue was provided by an immunohistochemical study carried out by Chen et al. ${ }^{8}$, which showed that the BRCA1 protein was normally found in the nucleus. This observation is now significantly extended by Chapman and Verma ${ }^{4}$, who demonstrate that the carboxy terminus of BRCA1 contains a transcriptional activation domain; this transcriptional activation capacity was markedly diminished in each of four mutant BRCA1 proteins derived from patients with hereditary breast cancer.

Chen et al. ${ }^{8}$ reported that the localization of BRCA1 was often aberrant (in the cell cytoplasm instead of the nucleus) in non-hereditary breast cancers. This observation was exciting, because it suggested that BRCA1 might play a role in nonhereditary breast cancers (which account for more than 90 per cent of the total breast cancers in the Western world) as well as in the hereditary types. But, alas, the excitement was short-lived, as other investigators, using similar immunohistochemical techniques, concluded that BRCA1 was sited in the nucleus in both normal tissues and non-hereditary breast cancers ${ }^{9}$.

Further complicating the idea that $B R C A 1$ encodes a transcription factor is a report suggesting that BRCA1 is not nuclear at all, but rather is a secreted protein localized in secretory vesicles ${ }^{10}$. This was provocative, in part because it might explain one of the most puzzling aspects of
BRCA1 epidemiology. Whereas inherited mutations of $B R C A 1$ lead to predisposition to breast and ovarian cancer, acquired $B R C A 1$ mutations are extraordinarily rare in sporadic (non-hereditary) breast or ovarian cancers. This is in stark contrast to most previously described tumour-suppressor genes, in which somatic mutations result in sporadic cancers of the same type.

This conundrum might be explicable if BRCA1 were secreted, thereby precluding a cell-autonomous effect on neoplasia. However, because breast lobules normally develop in a monoclonal fashion ${ }^{11}$, one can imagine that a somatic mutation of $B R C A 1$ could initiate neoplasia if it occurred in a lobule with a pre-existing hereditary mutation of the other allele.

These conflicting results illustrate the power of knockout mice to resolve fundamental questions about cancer genes. For example, nuclear (cell-autonomous) and secreted (non-autonomous) models of BRCA1 function predict dramatically different phenotypes in experimental situations, particularly with chimaeras between wild-type and knockout mouse embryonic stem cells. Additionally, a major problem for cell localization studies is that an antibody's specificity as indicated by immunohistochemistry cannot necessarily be inferred from its reactivity in western blots or immunoprecipitation ${ }^{12}$.

What is needed to guarantee specificity are perfectly matched controls, in which endogenous levels of the relevant protein can be assessed immunohistochemically. Mouse cells with disrupted $B R C A 1$ genes can provide such controls: absolutely no reactivity with the antibody should be detected in such cells, in contrast to otherwise isogeneic cells of the same subtype. Although analogous controls may be furnished by breast tumour cells from humans with hereditary $B R C A 1$ mutations ${ }^{13}$, the mice provide a more reproducible and manageable source of such reagents. With the availability of mouse knockouts, it therefore should not be long until we know where the $B R C A 1$ gene product really lives, at least in mice.

Kenneth W. Kinzler and Bert Vogelstein are in the Johns Hopkins Oncology Center and Howard Hughes Medical Institute, $424 \mathrm{~N}$. Bond Street, Baltimore, Maryland 21231, USA.

\footnotetext{
1. Miki, Y. et al. Science 266, 66-71 (1994).

2. Gowen, L. C. et al. Nature Genet. 12, 191-194 (1996)

3. Hakem, R. et al. Cell 85, 1009-1023 (1996).

4. Chapman, M. S. \& Verma, I. M. Nature 382, 678-679 (1996).

5. Boyd, M., Harris, F., McFarlane, R., Davidson, H. R. \& Black, D. M. Nature 375, 541-542 (1995).

6. Vaughn, J. P. et al. Cell Growth Different. 7, 711-715 (1996).

7. Gudas, J. M. et al. Cell Growth Different. 7, 717-723 (1996).

8. Chen, Y. et al. Science 270, 789-791 (1995).

9. Scully, R. et al. Science 272, 123-125 (1996).

10. Jensen, R. A et al Nature Genet. 12, 303-308 (1996).

11. Tsai, Y. C. et al. Cancer Res. 56, 402-404 (1996).

12. Wilson, C. A. et al. Nature Genet. 13, 264-265 (1996).

13. Jensen, R. A. et al. Nature Genet. 13, 269-272 (1996).
}

NATURE · VOL 382 · 22 AUGUST 1996 